\title{
An unusual presentation a spontaneous lung pseudocyst that mimicked a congenital lobar over-inflation
}

\author{
Meryline Ndlovu ${ }^{1}$, Refiloe Masekela ${ }^{2}$, Stanley Thula ${ }^{2}$, and Prakash Jeena ${ }^{2}$ \\ ${ }^{1}$ University of KwaZulu-Natal \\ ${ }^{2}$ University of KwaZulu-Natal College of Health Sciences
}

September 11, 2020

An unusual presentation of a spontaneous lung pseudocyst that mimicked a congenital lobar over-inflation

M Ndlovu, Cert Pulm (SA) Paed; S A Thula, Cert Pulm (SA) Paed; R Masekela, (PhD); P M Jeena, $\mathrm{PhD}$.

Department of Paediatrics and Child Health, Nelson $R$ Mandela School of Clinical Medicine, College of Health Sciences, University of KwaZulu-Natal, Durban, South Africa

Corresponding author: M Ndlovu (meendlovu@gmail.com)

A pulmonary pseudocyst is defined as a cyst that lacks respiratory epithelial cells. Lung pseudocysts are rare lesions in children and their classification is usually combined with other parenchymal cystic lesions. The distinction between a pseudo and a true cyst is difficult to distinguish both in utero and postnatally. Radiological investigations such as x-ray and computed tomography (CT) are equally non-specific. Most literature focuses on pseudocysts in adults particularly following blunt or penetrating chest trauma.

K. X was a 2 year 5 months old male who presented to the district hospital with shortness of breath and mild chest retractions since he was 8 months old. There was no history of trauma to the chest. He had been seen at the local clinic on two occasions and was managed as a pneumonia with oral antibiotics. There was no resolution of symptoms, but the caregiver did not seek further care because the child was active as she had been advised that the child would improve with age. At the 18-month immunisations follow up visit, he was noted to have growth faltering and was placed on nutritional supplements. The shortness of breath progressively worsened and they presented to the district hospital where the child was admitted. He was noted to be in moderate respiratory distress, emaciated with an oxygen saturation of $88 \%$ in room air that improved to $100 \%$ on 2L oxygen via nasal canula. In addition, he had clubbing, with a symmetrically hyperinflated chest.

A CXR at the district hospital revealed a hyperlucent right lung with mediastinal shift to the contralateral side. (Fig 1) A diagnosis of right sided pneumothorax was made, and an intercostal chest drain (ICD) was inserted. There was no clinical or radiological improvement after ICD insertion and a suspicion of congenital lobar over-inflation was made. The patient was then transferred to a tertiary hospital for paediatric pulmonology assessment where he was noted to be in moderate respiratory distress with markedly reduced breath sounds bilaterally. The ICD had slipped out during transfer, but the patient's clinical condition had remained stable. 


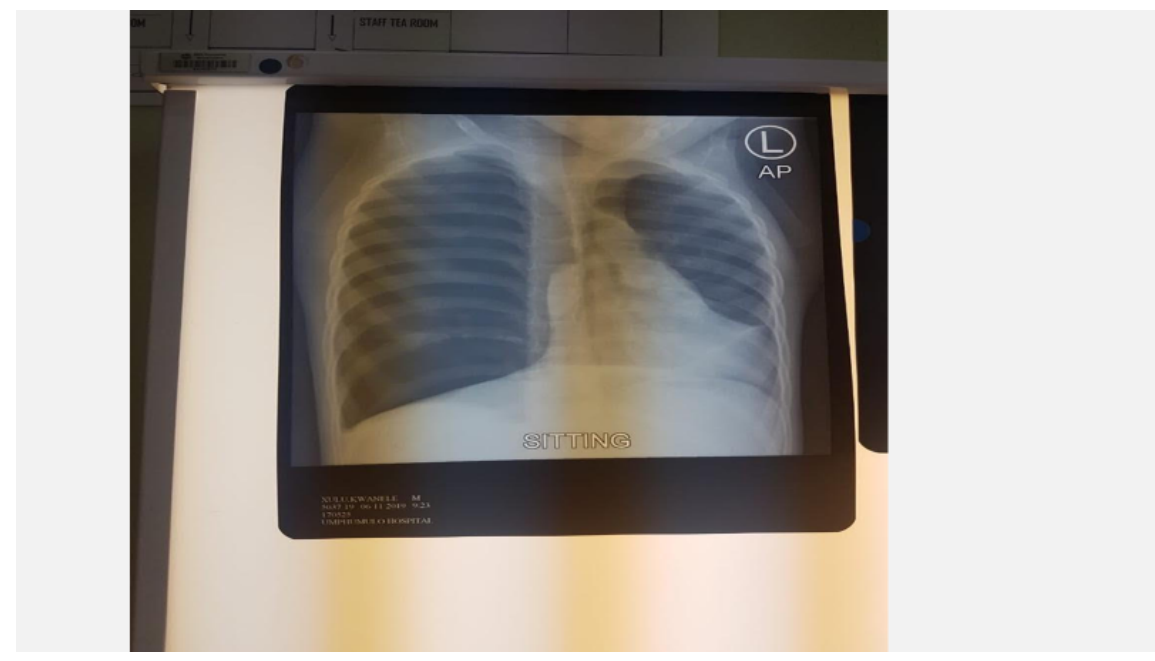

Fig. 1: First CXR showing right hyperlucency with left-ward herniation and compression of left lung.

A new chest x-ray showed the previously noted hyperlucency occupying the entire right hemithorax and herniating to the left and compressing the left lung. (Fig 2) The trachea was deviated to the left and no lung markings were visible on the right. The differential diagnoses included congenital pulmonary airway malformation (CPAM), congenital lobar over inflation (CLO) and vascular sling. Acquired causes like foreign body and extrinsic lymph node compression, with ball-valve effect and unilateral airway trapping were also entertained. A contrasted CT confirmed the right-sided hyperlucent lung with herniation to the left and left tracheal deviation. In addition, a small compressed right lung could be visualised on the axial lung field view. (Fig 3) The left lung was also compressed. Mediastinal lymph nodes and vascular rings were excluded on mediastinal windows. His blood work-up was unremarkable.

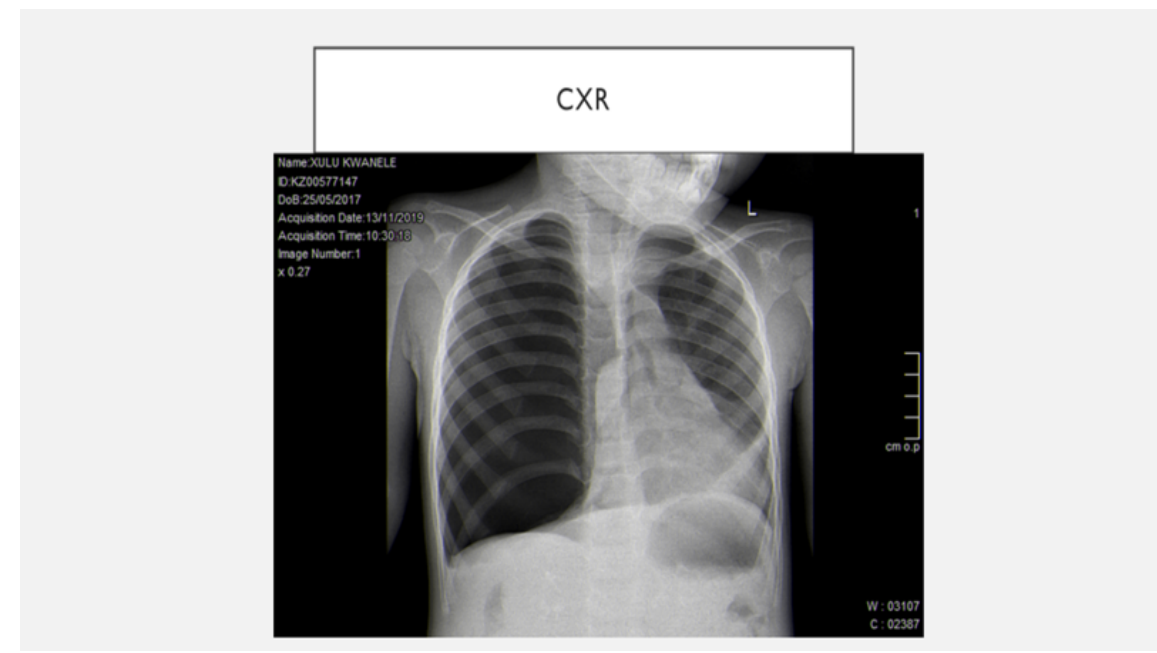

Fig 2. Right sided hyperlucency herniating to the left and tracheal deviation to the left 


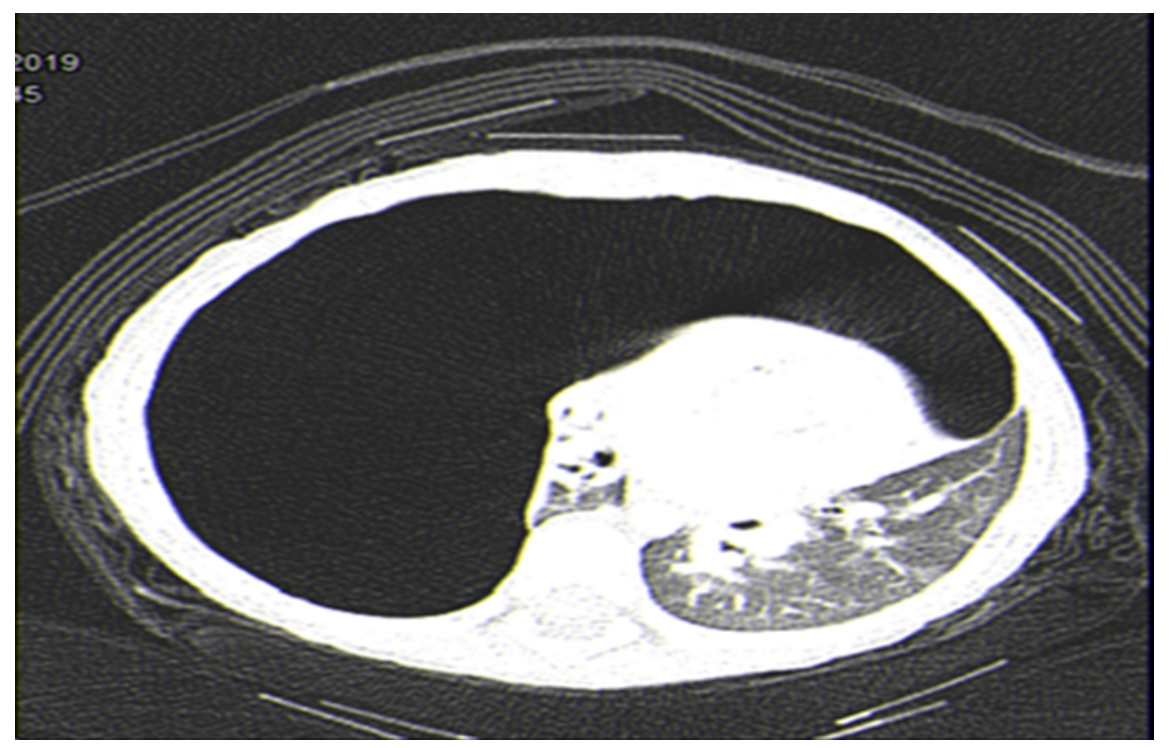

Fig 3. Axial slice showing hyperlucency with a very small compressed right lung and a small left lung.

A ventilation- perfusion scan showed decreased ventilation and perfusion of the right lung in comparison with the left lung. (Fig 4) The patient was taken to theatre by the cardiothoracic surgeons who performed a right postero-lateral thoracotomy and found a large air-filled cyst arising from the right lower lobe. The cyst had a small neck connecting it to the right lower lobe. The cyst was excised, and the right lung lobes immediately re-expanded on ventilation of the right bronchus. A rigid bronchoscopy performed during the same setting revealed no airway abnormalities. Post operatively, the patient was ventilated for 2 days and there were no complications. Histology showed a cyst devoid of epithelial or inflammatory cells which was consistent with a pseudocyst. The patient had a good outcome and was discharged home. A month later he was reviewed, and a repeat chest radiograph was satisfactory. (Fig 5)

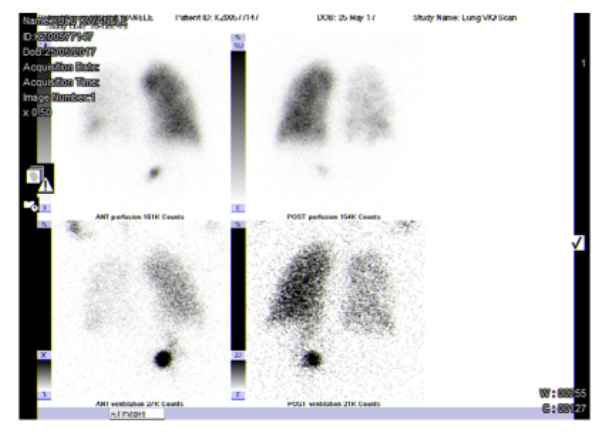

Fig 4. Ventilation/perfusion scan demonstrating decreased uptake on the right lung in comparison with the left. 


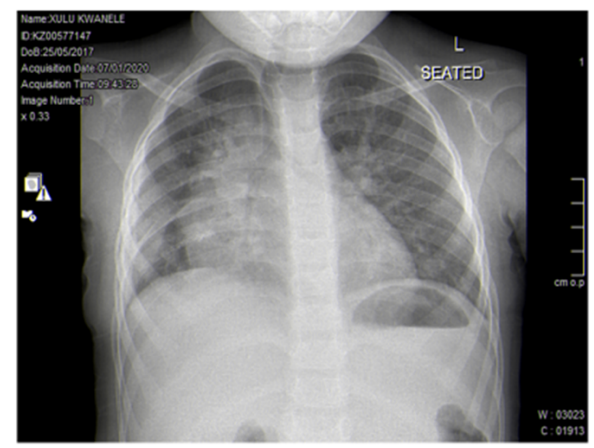

Fig 5. CXR on follow-up showing re-expansion of the right lung

Pulmonary pseudocysts are often a sequela of blunt trauma to the chest wall, although cases following penetrating injuries to the chest have also been described. (1) Traumatic pulmonary pseudocysts often occur within 24 hours after the injury, may be single or multiple and are usually self-limiting with an excellent prognosis. ${ }^{(2)}$ Multiple pseudocysts not related to trauma have been reported in neonates with hyaline membrane disease on mechanical ventilation. ${ }^{(3)}$ Pseudocysts with predominant inflammatory processes are usually multiple, small and have been described in smokers, asthmatics and in cystic fibrosis patients. ${ }^{(4)}$. Some authors have lumped pseudocysts with congenital cystic lung lesions under a broader group of cystic and pseudocystic pulmonary malformations. (CPPM) ${ }^{(5)}$ Most CPPM lesions disappear in utero and are generally asymptomatic but may be responsible for fetal death, early life respiratory distress, recurrent pulmonary infections, or can transform to malignant lesions. ${ }^{(5)}$ It is challenging to make a distinction between cystic and pseudocystic pulmonary lesions radiologically, on chest radiography or HRCT. Definitive diagnosis of a pseudocyst is only possible histopathologically, where the absence of epithelial cells or lung tissue is diagnostic of a pseudocyst. The management of congenital pulmonary airway malformation in asymptomatic children is controversial, some adopt a wait-and- see approach while others prefer a more aggressive approach of surgical excision to avert the risk for malignant transformation. In symptomatic patients, it is prudent to excise the cyst to achieve symptom relief.

1. Gulbahar G. Diagnosing traumatic pulmonary pseudocyst. South Med J. 2009; 102(9):881. doi : 10.1097/SMJ.0b013e3181b187d3.

2. Kanj A, Tabaja H, Soubani AO, Kanj N. Traumatic Pulmonary Pseudocyst Mimicking a Congenital Cystic Lung Disease. Case reports in pulmonology. 2018; 2018:7269694.doi : 10.1155/2018/7269694

3. Harris H. Pulmonary pseudocysts in the newborn infant. Pediatrics. 1977; 59(2):199-204. doi : 10.1136/adc.58.8.612.

4. Tomashefski JF Jr, Bruce M, Stern RC, Dearborn DG, Dahms B. Pulmonary air cysts in cystic fibrosis: relation of pathologic features to radiologic findings and history of pneumothorax. Hum Pathol 1985; 16: 253-261.doi: 10.1016/s0046-8177(85)80011-3

5. Rodríguez-Velasco A, López-Jara-Zárate EJ, Vargas MH, Ramírez-Figueroa JL, Furuya ME. Cystic and pseudocystic pulmonary malformations in children: Clinico-pathological correlation. Ann Diagn Pathol. 2019; 39:78-85. doi : 10.1016/j.anndiagpath.2019.02.005 